Graziano Crasta

\title{
A symmetry problem in the calculus of variations
}

Received October 22, 2004 and in revised form March 29, 2005

Abstract. We consider the integral functional

$$
J(u)=\int_{\Omega}[f(|D u|)-u] d x, \quad u \in W_{0}^{1,1}(\Omega),
$$

where $\Omega \subset \mathbb{R}^{n}, n \geq 2$, is a nonempty bounded connected open subset of $\mathbb{R}^{n}$ with smooth boundary, and $\mathbb{R} \ni s \mapsto f(|s|)$ is a convex, differentiable function. We prove that if $J$ admits a minimizer in $W_{0}^{1,1}(\Omega)$ depending only on the distance from the boundary of $\Omega$, then $\Omega$ must be a ball.

Keywords. Minimizers of integral functionals, distance function, Euler equation

\section{Introduction}

Let $\Omega \subset \mathbb{R}^{n}, n \geq 2$, be a smooth domain, and let $J_{2}$ be the integral functional defined in $H_{0}^{1}(\Omega)$ by

$$
J_{2}(u)=\int_{\Omega}\left(\frac{1}{2}|D u|^{2}-u\right) d x, \quad u \in H_{0}^{1}(\Omega) .
$$

It is well known that $J_{2}$ has a unique minimum point in $H_{0}^{1}(\Omega)$, which is the unique solution of the Dirichlet problem

$$
\begin{cases}-\Delta u=1 & \text { in } \Omega \\ u=0 & \text { on } \partial \Omega\end{cases}
$$

In recent papers [11, 10], the following question arises in connection with the estimate of the minimum of $J_{2}$ : If the minimizer $u_{0}$ of $J_{2}$ depends only on the distance from the boundary of $\Omega$, what can be said about the geometry of $\Omega$ ?

G. Crasta: Dipartimento di Matematica "G. Castelnuovo", Università di Roma I,

P.le A. Moro 2, 00185 Roma, Italy; e-mail: crasta@mat.uniroma1.it

Mathematics Subject Classification (2000): Primary 49K30; Secondary 49K20, 49K24, 53A07 
In order to answer to this question, let us recall a celebrated result by J. Serrin (see [19]) which states that if the overdetermined Dirichlet problem

$$
\begin{cases}-\Delta u=1 & \text { in } \Omega \\ u=0 & \text { on } \partial \Omega \\ \left|\frac{\partial u}{\partial v}\right|=c & \text { on } \partial \Omega\end{cases}
$$

admits a solution, then $\Omega$ must be a ball. (Here $\partial u / \partial v$ denotes the derivative with respect to the outer normal to the boundary of $\Omega$.)

Now, assume that the minimizer $u_{0}$ of $J_{2}$ depends only on the distance $d_{\Omega}$ from the boundary of $\Omega$, that is, $u_{0}(x)=\phi\left(d_{\Omega}(x)\right), x \in \Omega$. Since the outer normal to $\partial \Omega$ is given by $v(y)=-D d_{\Omega}(y)$ for every $y \in \partial \Omega$, we have

$$
\left|\frac{\partial u_{0}}{\partial v}(y)\right|=\left|\phi^{\prime}(0) D d_{\Omega}(y) \cdot v(y)\right|=\left|\phi^{\prime}(0)\right|, \quad \forall y \in \partial \Omega,
$$

hence $u_{0}$ is a solution to the overdetermined problem 22 with $c=\left|\phi^{\prime}(0)\right|$. From Serrin's result, we conclude that $\Omega$ is a ball.

We remark that the above argument works also for the functional

$$
J_{p}(u)=\int_{\Omega}\left(\frac{1}{p}|D u|^{p}-u\right) d x, \quad u \in W_{0}^{1, p}(\Omega), 1<p<\infty,
$$

using the analog of Serrin's result for the $p$-Laplace operator (see [3, 13, 12]).

Our aim is to extend this kind of symmetry results to the general functional

$$
J(u)=\int_{\Omega}[f(|D u|)-u] d x, \quad u \in W_{0}^{1,1}(\Omega),
$$

where $\Omega \subset \mathbb{R}^{n}$ is a smooth domain and $\mathbb{R} \ni s \mapsto f(|s|)$ is a convex, differentiable function. Let us define the set of so-called web functions (or radial functions)

$$
\mathcal{W}(\Omega)=\left\{u \in W_{0}^{1,1}(\Omega) ; u \text { depends only on the distance from } \partial \Omega\right\} .
$$

We prove that if the minimum problem

$$
\min \left\{J(u) ; u \in W_{0}^{1,1}(\Omega)\right\}
$$

admits a solution belonging to $\mathcal{W}(\Omega)$, then $\Omega$ is a ball (see Theorem 1 ). The converse is also true (see Remark 1).

Our approach is not based on the analysis of an associated overdetermined problem. Indeed, the Euler equation associated to the functional $J$ is, at least formally, the nonlinear Dirichlet problem

$$
\begin{cases}-\operatorname{div}\left(f^{\prime}(|D u|) \frac{D u}{|D u|}\right)=1 & \text { in } \Omega, \\ u=0 & \text { on } \partial \Omega .\end{cases}
$$


For such general equations there are no results concerning the overdetermined problem with

$$
\left|\frac{\partial u}{\partial v}\right|=c \quad \text { on } \partial \Omega
$$

similar to Serrin's result for (2).

On the other hand, the requirement that the integral functional $J$ has a minimizer in $\mathcal{W}(\Omega)$ is much stronger than the boundary condition $(7)$. We shall fully exploit this assumption in order to obtain the following results.

(a) An explicit representation formula for the solutions to the minimum problem

$$
\min \{J(u) ; u \in \mathcal{W}(\Omega)\}
$$

(see Theorem 2 below).

(b) The validity in the sense of distributions of the Euler-Lagrange equation (6) associated to the minimum problem $[5$, for minima belonging to $\mathcal{W}(\Omega)$ (see Theorem 3 below).

(c) Existence, uniqueness and explicit representation of the solution $v$ of the equation

$$
-\operatorname{div}\left(v(x) D d_{\Omega}(x)\right)=1 \quad \text { in } \Omega,
$$

which is related to the Euler-Lagrange equation (6) when $u \in \mathcal{W}(\Omega)$.

We remark that the results in (c) were obtained in [5,6] in the framework of mathematical models for sandpile growth.

Combining (a), (b) and (c) we shall prove that the mean curvature of $\partial \Omega$ is constant. This implies that $\Omega$ must be a ball, thanks to a fundamental result in differential geometry by A. D. Aleksandrov [1].

The paper is organized as follows. In Section 2 we state the main result of the paper (see Theorem 1 below). The existence and characterization of minimizers of $J$ in the space $\mathcal{W}(\Omega)$ is established in Section 3 , whereas the validity of the Euler-Lagrange equation for minimizers of $J$ in $W_{0}^{1,1}(\Omega)$, belonging to $\mathcal{W}(\Omega)$, is proven in Section 4 Finally, in Section 5 we recall the result proven in [6] and complete the proof of Theorem 1 .

\section{Notation and statement of the result}

In what follows, $\Omega$ will denote a smooth domain in $\mathbb{R}^{n}$, that is, a nonempty bounded open connected subset of $\mathbb{R}^{n}$ with $C^{2}$ boundary. We denote by $\partial \Omega$ the boundary of $\Omega$, and by $d_{\Omega}: \bar{\Omega} \rightarrow \mathbb{R}$ the distance function to $\partial \Omega$. The symbol $r_{\Omega}$ will denote the inradius of $\Omega$, that is, the supremum of the radii of the balls contained in $\Omega$. It is easily seen that $r_{\Omega}=\max \left\{d_{\Omega}(x) ; x \in \Omega\right\}$.

If $A \subset \mathbb{R}^{n}$, we denote by $|A|$ and $\mathcal{H}^{n-1}(\partial A)$, respectively, the Lebesgue measure of $A$ and the $(n-1)$-dimensional Hausdorff measure of $\partial A$.

Let $J$ be the functional defined in (3). Our assumptions on $f$ are the following: 
(F1) $f:[0, b) \rightarrow \mathbb{R}, b \in(0, \infty]$, is a convex, differentiable, nondecreasing function. (If $b<\infty$, for notational convenience we set $f(s)=\infty$ for every $s \geq b$.)

(F2) If $0<b<\infty$,

$$
\lim _{s \rightarrow b^{-}} f(s)=\infty
$$

if $b=\infty$,

$$
\lim _{s \rightarrow \infty} \frac{f(s)}{s}=\lim _{s \rightarrow \infty} f^{\prime}(s)>\frac{1}{n}\left(\frac{|\Omega|}{v_{n}}\right)^{1 / n},
$$

where $v_{n}$ is the volume of the unit ball in $\mathbb{R}^{n}$.

(F3) $f_{+}^{\prime}(0):=\lim _{s \rightarrow 0^{+}} \frac{f(s)-f(0)}{s}=0$.

We remark that, in the case $b=\infty$, assumption (F2) is certainly satisfied if $f$ is a superlinear function, that is, if

$$
\lim _{s \rightarrow \infty} \frac{f(s)}{s}=\infty
$$

Assumption (F3) is equivalent to the differentiability of the map $s \mapsto f(|s|)$ at $s=0$.

Our main result is the following.

Theorem 1. Let $\Omega \subset \mathbb{R}^{n}$ be a smooth domain, let $J$ be the functional defined in (3), and assume that $f$ satisfies assumptions $(\mathrm{F} 1)-(\mathrm{F} 3)$. If $J$ admits a minimizer in $W_{0}^{1,1}(\Omega)$ that depends only on the distance from the boundary of $\Omega$, then $\Omega$ must be a ball.

The proof of this theorem is postponed to Section 5

Remark 1. Of course, the converse of Theorem 1 also holds. Namely, if $\Omega$ is a ball and $f$ satisfies assumptions (F1)-(F3), then the functional $J$ admits a unique minimizer in $W_{0}^{1,1}(\Omega)$, which is radially symmetric (see for example [8]).

Remark 2. In Theorem 1 , the assumption that $f$ be differentiable cannot be dropped. Namely, let $f(s)=\max \{0, s-\rho\}, s \geq 0$, where $\rho \geq 0$ is a fixed parameter. Then $f$ is a convex nondecreasing function in $[0, \infty)$, and, if $\rho>0$, it is not differentiable at $s=\rho$. Let $\Omega \subset \mathbb{R}^{n}$ be a smooth domain. Assume in addition that $\Omega$ is a convex set, and that $|\Omega| \leq v_{n}$, so that (F2) clearly holds. Under these assumptions, in [20] it was proven that the function $u_{0}(x)=\rho d_{\Omega}(x)$ is a minimizer of $J$ in $W_{0}^{1,1}(\Omega)$. (In [6] the same result is proven also in the case of nonconvex domains.) This assertion also holds in the case $\rho=0$. We remark that, in this case, $f$ does not satisfy assumption (F3). This example shows that, for such $f$, the functional $J$ admits minimizers depending only on the distance from $\partial \Omega$ even if $\Omega$ is not a ball. 


\section{Existence of minimizers in $\mathcal{W}(\Omega)$}

The aim of this section is to prove, under the assumptions of Theorem 1 , that the functional $J$ has a minimizer in the space $\mathcal{W}(\Omega)$ of web functions. Moreover, we give an explicit representation of the minimizers and we prove that they satisfy a suitable EulerLagrange inclusion.

Throughout this section, $\Omega$ will be a smooth domain of $\mathbb{R}^{n}$, although Theorem 2 below still holds under a weaker regularity assumption on the boundary of $\Omega$ (see Remark 4 below).

We recall that $d_{\Omega}$ is a Lipschitz continuous function, with gradient satisfying $\left|D d_{\Omega}(x)\right|=1$ for a.e. $x \in \Omega$. It is clear from the definition that $0 \leq d_{\Omega}(x) \leq r_{\Omega}$ for every $x \in \bar{\Omega}$, where $r_{\Omega}$ denotes the inradius of $\Omega$. For every $i=1, \ldots, n-1$, denote by $\kappa_{i}(y)$ the $i$-th principal curvature of $\partial \Omega$ at the point $y \in \partial \Omega$, corresponding to a principal direction $e_{i}(y)$ orthogonal to $D d_{\Omega}(y)$, with the sign convention $\kappa_{i}(y) \geq 0$ if the normal section of $\Omega$ along the direction $e_{i}$ is convex. Let $\Sigma$ denote the singular set of $d_{\Omega}$, that is, the set of points $x \in \Omega$ for which $d_{\Omega}$ is not differentiable. The set $\bar{\Sigma}$ is also known as ridge or cut locus. From Rademacher's theorem, $\Sigma$ has vanishing $n$ dimensional Lebesgue measure. Introducing the projection $\Pi(x)$ of $x \in \Omega$ on $\partial \Omega, \Sigma$ is also the set of points $x$ for which $\Pi(x)$ is not a singleton. We extend $\kappa_{i}, i=1, \ldots, n-1$, to $\bar{\Omega} \backslash \bar{\Sigma}$ by setting $\kappa_{i}(x)=\kappa_{i}(\Pi(x))$ for every $x \in \Omega \backslash \bar{\Sigma}$. Define the normal distance to the cut locus of $\Omega$ by

$$
\tau(x)= \begin{cases}\min \left\{t \geq 0 ; x+t D d_{\Omega}(x) \in \bar{\Sigma}\right\} & \text { if } x \in \bar{\Omega} \backslash \bar{\Sigma} \\ 0, & \text { if } x \in \bar{\Sigma}\end{cases}
$$

It is known that if $\partial \Omega$ is of class $C^{2,1}$, then $\tau$ is Lipschitz continuous on $\partial \Omega$ (see [18, 17]); for less regular domains the Lipschitz continuity may fail, but continuity is preserved if $\partial \Omega$ is of class $C^{2}$ (see [5, 6]).

The function $f:[0, \infty) \rightarrow[0, \infty]$ appearing in the definition of $J$ will be a lower semicontinuous, nondecreasing convex function. We remark that, for the results established in this section, $f$ need not be differentiable, and can take the value $\infty$. With some abuse of notation, we will denote by $f^{*}: \mathbb{R} \rightarrow \mathbb{R} \cup\{\infty\}$ the conjugate function of the map $s \mapsto f(|s|), s \in \mathbb{R}$. As is customary, the symbol $\partial f(s)$ will denote the subgradient of $f$ at $s$, in the sense of convex analysis. by

In the following, a major role will be played by the function $\alpha:\left[0, r_{\Omega}\right] \rightarrow \mathbb{R}$ defined

$$
\alpha(t)= \begin{cases}\frac{\left|\Omega_{t}\right|}{\mathcal{H}^{n-1}\left(\partial \Omega_{t}\right)} & \text { if } t \in\left[0, r_{\Omega}\right), \\ 0 & \text { if } t=r_{\Omega},\end{cases}
$$

where $\Omega_{t}=\left\{x \in \Omega ; d_{\Omega}(x)>t\right\}$. (We recall that $r_{\Omega}$ denotes the inradius of $\Omega$.)

Theorem 2. Let $\Omega$ be a smooth domain in $\mathbb{R}^{n}$. Let $J$ be the functional defined in (3), where $f:[0, \infty) \rightarrow[0, \infty]$ is a lower semicontinuous, nondecreasing convex function, satisfying

$$
f(s) \geq M s-a, \quad \forall s \geq 0,
$$


for some positive constants $M$ and $a$, with

$$
M>\frac{1}{n}\left(\frac{|\Omega|}{v_{n}}\right)^{1 / n}
$$

Then, for every measurable selection

$$
\gamma(t) \in \partial f^{*}(\alpha(t)), \quad t \in\left[0, r_{\Omega}\right]
$$

the function

$$
u_{0}(x)=\int_{0}^{d_{\Omega}(x)} \gamma(t) d t, \quad x \in \Omega,
$$

belongs to $W^{1, \infty}(\Omega)$ and is a minimizer of $J$ in the set $\mathcal{W}(\Omega)$ defined in $(4)$. Conversely, if $u_{0} \in \mathcal{W}(\Omega)$ is a minimizer of $J$ in $\mathcal{W}(\Omega)$, then $u_{0}$ belongs to $W^{1, \infty}(\Omega)$ and satisfies the Euler-Lagrange inclusion

$$
\left|D u_{0}(x)\right| \in \partial f^{*}\left(\alpha\left(d_{\Omega}(x)\right)\right), \quad \text { a.e. } x \in \Omega .
$$

Remark 3. Under the additional assumption of convexity of $\Omega$, Theorem 2 was proved in [9], without convexity assumptions on $f$.

The remaining part of this section will be devoted to the proof of Theorem 2 We start by proving a simple estimate on the function $\alpha$ defined in 9 .

Lemma 1. For every $t \in\left[0, r_{\Omega}\right)$ we have

$$
0<\alpha(t) \leq \frac{1}{n}\left(\frac{\left|\Omega_{t}\right|}{v_{n}}\right)^{1 / n} \leq \frac{1}{n}\left(\frac{|\Omega|}{v_{n}}\right)^{1 / n} .
$$

(Recall that $v_{n}$ is the volume of the unit ball in $\mathbb{R}^{n}$.) As a consequence, $\lim _{t \rightarrow r_{\Omega}} \alpha(t)$ $=\alpha\left(r_{\Omega}\right)=0$.

Proof. The fact that $\alpha$ is a positive function on $\left[0, r_{\Omega}\right)$ follows from its very definition. Concerning the upper bound, we recall that the isoperimetric inequality

$$
\mathcal{H}^{n-1}(\partial A) \geq n v_{n}^{1 / n}|A|^{(n-1) / n}
$$

holds for every bounded measurable set $A \subset \mathbb{R}^{n}$ (see [4, §14.3 and §14.6]). Applying (15) to the bounded measurable set $\Omega_{t}$ we get

$$
0<\alpha(t)=\left|\Omega_{t}\right|^{1 / n} \frac{\left|\Omega_{t}\right|^{(n-1) / n}}{\mathcal{H}^{n-1}\left(\partial \Omega_{t}\right)} \leq \frac{1}{n v_{n}^{1 / n}}\left|\Omega_{t}\right|^{1 / n} \leq \frac{1}{n}\left(\frac{|\Omega|}{v_{n}}\right)^{1 / n}
$$

and the proof is complete. 
Let us define the set

$$
\mathcal{K}=\left\{\begin{array}{l|l}
\phi \in A C_{\mathrm{loc}}\left[0, r_{\Omega}\right) & \begin{array}{l}
\phi(0)=0 \\
t \mapsto \mathcal{H}^{n-1}\left(\partial \Omega_{t}\right) \phi(t) \in L^{1}\left(0, r_{\Omega}\right) \\
t \mapsto \mathcal{H}^{n-1}\left(\partial \Omega_{t}\right) \phi^{\prime}(t) \in L^{1}\left(0, r_{\Omega}\right)
\end{array}
\end{array}\right\}
$$

where $A C_{\text {loc }}\left[0, r_{\Omega}\right)$ denotes the set of absolutely continuous functions in $[0, r]$ for every $r \in\left(0, r_{\Omega}\right)$.

Lemma 2. A function $u$ belongs to $\mathcal{W}(\Omega)$ if and only if $u=\phi \circ d_{\Omega}$ for some $\phi \in \mathcal{K}$.

Proof. Let $u \in \mathcal{W}(\Omega)$. By definition of $\mathcal{W}(\Omega)$, there exists a measurable function $\phi:\left[0, r_{\Omega}\right] \rightarrow \mathbb{R}$ such that $u(x)=\phi\left(d_{\Omega}(x)\right)$ for every $x \in \Omega$.

The tricky part of the proof that $\phi \in \mathcal{K}$ consists in showing that $\phi$ belongs to $A C_{\text {loc }}\left[0, r_{\Omega}\right)$. We shall use a local coordinate system in $\Omega$ whose properties were proved in [16, p. 236]. More precisely, since $\Omega$ has $C^{2}$ boundary, we can choose a finite family $\mathcal{U}_{1}, \ldots, \mathcal{U}_{N}$ of bounded open sets in $\mathbb{R}^{n}$ so that $\partial \Omega \subseteq \bigcup_{i=1}^{N} \mathcal{U}_{i}$, and such that, for each $i=1, \ldots, N$, in a suitable coordinate system in $\mathbb{R}^{n}$ we have

$$
\Omega \cap \mathcal{U}_{i}=\left\{\left(x^{\prime}, t\right) ; x^{\prime} \in V_{i}, t>\Phi_{i}\left(x^{\prime}\right)\right\} \cap \mathcal{U}_{i},
$$

where $V_{i}=\left\{x^{\prime} \in \mathbb{R}^{n-1} ;\left(x^{\prime}, t\right) \in \mathcal{U}_{i}\right.$ for some $\left.t \in \mathbb{R}\right\}$ is an open set, and $\Phi_{i}$ is a $C^{2}$ function on $\mathbb{R}^{n-1}$. Define the maps $G_{i}: V_{i} \times \mathbb{R} \rightarrow \mathbb{R}^{n}, i=1, \ldots, N$, by

$$
G_{i}\left(x^{\prime}, t\right)=y+t D d_{\Omega}^{s}(y), \quad \text { where } y=\left(x^{\prime}, \Phi_{i}\left(x^{\prime}\right)\right) \in \partial \Omega,
$$

and $d_{\Omega}^{S}$ denotes the signed distance to $\partial \Omega$ defined by

$$
d_{\Omega}^{s}(x)= \begin{cases}\operatorname{dist}(x, \partial \Omega) & \text { if } x \in \Omega \\ -\operatorname{dist}(x, \partial \Omega) & \text { if } x \in \mathbb{R}^{n} \backslash \Omega\end{cases}
$$

We collect here the main properties of the maps $G_{i}, i=1, \ldots, N$ (see [16, Lemmas 14 and 15]):

(a) $G_{i}$ is Lipschitz continuous on bounded subsets of $V_{i} \times \mathbb{R}$.

(b) The Jacobian $J G_{i}$ is a locally bounded measurable function, and

$$
J G_{i}\left(x^{\prime}, t\right)=\sqrt{1+\left|D \Phi_{i}\left(x^{\prime}\right)\right|^{2}} \prod_{j=1}^{n-1}\left(1-\kappa_{j} t\right),
$$

where $\kappa_{1}, \ldots, \kappa_{n-1}$ are the principal curvatures of $\partial \Omega$ at $G_{i}\left(x^{\prime}, 0\right)$.

(c) $G_{i}$ is one-to-one on the set

$$
U_{i}=\left\{\left(x^{\prime}, t\right) ; x^{\prime} \in V_{i}, t \in\left(0, \tau\left(G_{i}\left(x^{\prime}, 0\right)\right)\right)\right\} \subset \mathbb{R}^{n} .
$$

(d) $\Omega \backslash \Sigma=\bigcup_{i=1}^{N} G_{i}\left(U_{i}\right)$. 
From properties (a), (b) and (c) we deduce that, for every $i=1, \ldots, N$ and every $\epsilon>0$, $G_{i}$ is a bi-Lipschitz map on the set

$$
U_{i}^{\epsilon}=\left\{\left(x^{\prime}, t\right) ; x^{\prime} \in V_{i}, t \in\left(0, \max \left\{0, \tau\left(G_{i}\left(x^{\prime}, 0\right)\right)-\epsilon\right\}\right)\right\} .
$$

Since the restriction of $u$ to the set $A_{i}^{\epsilon}=G_{i}\left(U_{i}^{\epsilon}\right)$ belongs to $W^{1,1}\left(A_{i}^{\epsilon}\right)$, from Theorem 2.2.2 in [21] we see that the restriction of the composite map $v=u \circ G_{i}$ to $U_{i}^{\epsilon}$ belongs to $W^{1,1}\left(U_{i}^{\epsilon}\right)$.

Finally, let us prove that, for every fixed $\epsilon \in\left(0, r_{\Omega}\right)$, the map $\phi$ belongs to $A C[0$, $\left.r_{\Omega}-\epsilon\right]$. From property (d) and the fact that the $n$-dimensional Lebesgue measure of $\Sigma$ is zero, there exists an index $i \in\{1, \ldots, N\}$ such that

$$
\max \left\{\tau\left(G_{i}\left(x^{\prime}, 0\right)\right) ; x^{\prime} \in V_{i}\right\}=r_{\Omega} .
$$

From Theorem 2.1.4 in [21] we can assume that the function $v=u \circ G_{i}$ is absolutely continuous on the line segment

$$
\Lambda\left(x^{\prime}\right)=\left\{\left(x^{\prime}, t\right) ; t \in\left[0, \max \left\{0, \tau\left(G_{i}\left(x^{\prime}, 0\right)\right)-\epsilon / 2\right\}\right]\right\}
$$

for almost every $x^{\prime} \in V_{i}$. Since $\tau$ is a continuous map, there exists $x^{\prime} \in V_{i}$ such that $T=\tau\left(G_{i}\left(x^{\prime}, 0\right)\right)>r_{\Omega}-\epsilon$ and the restriction of $v$ to $\Lambda\left(x^{\prime}\right)$ is absolutely continuous. By the very definitions of the functions $G_{i}$ and $v$ we conclude that the map $t \mapsto u(y+$ $\left.t D d_{\Omega}(y)\right)=\phi(t)$, with $y=\left(x^{\prime}, \Phi_{i}\left(x^{\prime}\right)\right)$, is absolutely continuous in $[0, T]$; in particular, $\phi \in A C\left[0, r_{\Omega}-\epsilon\right]$. Furthermore, the boundary condition on $u$ implies that $\phi(0)=0$.

Let us conclude the proof of the lemma. From the change of variables formula (see [14, §3.4.3]) it follows that

$$
\begin{aligned}
\int_{\Omega}|u(x)| d x & =\int_{0}^{r_{\Omega}} \mathcal{H}^{n-1}\left(\partial \Omega_{t}\right)|\phi(t)| d t, \\
\int_{\Omega}|D u(x)| d x & =\int_{0}^{r_{\Omega}} \mathcal{H}^{n-1}\left(\partial \Omega_{t}\right)\left|\phi^{\prime}(t)\right| d t,
\end{aligned}
$$

hence $\phi \in \mathcal{K}$. Conversely, from the above formulas it is easily seen that if $\phi \in \mathcal{K}$, then $u=\phi \circ d_{\Omega}$ belongs to $W_{0}^{1,1}(\Omega)$.

Remark 4. Following [16, Section 3], it can be easily proved that the conclusion of Lemma 2 holds (with minor modifications in the proof) under a weaker assumption on the regularity of the boundary of $\Omega$. More precisely, it is enough that the bounded open set $\Omega \subset \mathbb{R}^{n}$ is of positive reach (see [15]), that is, there exists $r>0$ with the following property: for every $y \in \partial \Omega$ there exists a closed ball $B \subset \mathbb{R}^{n} \backslash \Omega$, of radius $r$, such that $B \cap \bar{\Omega}=\{y\}$.

As a consequence of Lemma 2, a function $u_{0}=\phi_{0} \circ d_{\Omega}$ is a minimizer of $J$ in $\mathcal{W}(\Omega)$ if and only if $\phi_{0}$ is a minimizer of the functional

$$
F(\phi)=\int_{0}^{r_{\Omega}} \mathcal{H}^{n-1}\left(\partial \Omega_{t}\right)\left[f\left(\left|\phi^{\prime}(t)\right|\right)-\phi(t)\right] d t
$$

in $\mathcal{K}$. In order to simplify the subsequent analysis, it is convenient to rewrite the term in $\phi$ using the following integration-by-parts formula. 
Lemma 3. If $\phi \in \mathcal{K}$, then the map $t \mapsto\left|\Omega_{t}\right| \phi^{\prime}(t)$ belongs to $L^{1}\left(0, r_{\Omega}\right)$ and

$$
\int_{0}^{r_{\Omega}}\left|\Omega_{t}\right| \phi^{\prime}(t) d t=\int_{0}^{r_{\Omega}} \mathcal{H}^{n-1}\left(\partial \Omega_{t}\right) \phi(t) d t .
$$

Proof. From the isoperimetric inequality $(15)$ we have

$$
\left|\Omega_{t}\right|=\left|\Omega_{t}\right|^{1 / n}\left|\Omega_{t}\right|^{(n-1) / n} \leq|\Omega|^{1 / n} \frac{1}{n v_{n}^{1 / n}} \mathcal{H}^{n-1}\left(\partial \Omega_{t}\right),
$$

hence

$$
\left|\Omega_{t}\right|\left|\phi^{\prime}(t)\right| \leq \frac{1}{n}\left(\frac{|\Omega|}{v_{n}}\right)^{1 / n} \mathcal{H}^{n-1}\left(\partial \Omega_{t}\right)\left|\phi^{\prime}(t)\right|, \quad \text { a.e. } t \in\left[0, r_{\Omega}\right] .
$$

Since $t \mapsto \mathcal{H}^{n-1}\left(\partial \Omega_{t}\right) \phi^{\prime}(t)$ belongs to $L^{1}\left(0, r_{\Omega}\right)$, so does $t \mapsto\left|\Omega_{t}\right| \phi^{\prime}(t)$. If we recall that $t \mapsto\left|\Omega_{t}\right|$ is absolutely continuous in $\left[0, r_{\Omega}\right]$ and $\frac{d}{d t}\left|\Omega_{t}\right|=-\mathcal{H}^{n-1}\left(\partial \Omega_{t}\right)$ for a.e. $t$, formula 20 now follows from a standard integration by parts.

In view of Lemma 3 , the functional $F$ can be rewritten as

$$
F(\phi)=\int_{0}^{r_{\Omega}} g\left(t, \phi^{\prime}(t)\right) d t, \quad \phi \in \mathcal{K},
$$

where $g:\left[0, r_{\Omega}\right] \times \mathbb{R} \rightarrow \mathbb{R} \cup\{\infty\}$ is defined by

$$
g(t, \xi)=\mathcal{H}^{n-1}\left(\partial \Omega_{t}\right) f(|\xi|)-\left|\Omega_{t}\right| \xi .
$$

The advantage of rewriting $F$ in this way lies in the fact that the lagrangean $g$ does not depend on $\phi$. We remark that $g(t, \cdot)$ is a convex function for every $t \in\left[0, r_{\Omega}\right]$. From assumption (10) and estimate (14) we have

$$
\begin{aligned}
g(t, \xi) & \geq \mathcal{H}^{n-1}\left(\partial \Omega_{t}\right)[M-\alpha(t)]|\xi|-\mathcal{H}^{n-1}\left(\partial \Omega_{t}\right) a \\
& \geq \mathcal{H}^{n-1}\left(\partial \Omega_{t}\right)\left[M-\frac{1}{n}\left(\frac{|\Omega|}{v_{n}}\right)^{1 / n}\right]|\xi|-\mathcal{H}^{n-1}\left(\partial \Omega_{t}\right) a,
\end{aligned}
$$

hence from the assumption 11 ,

$$
\lim _{|\xi| \rightarrow \infty} g(t, \xi)=\infty
$$

for every $t \in\left[0, r_{\Omega}\right)$. This implies that, for every such $t$, the convex function $g(t, \cdot)$ has a nonempty compact set of minimizers. Recalling that $\xi$ is a minimizer of $g(t, \cdot)$ if and only if $0 \in \partial g(t, \xi)$, that is, if and only if $\xi \in \partial g^{*}(t, 0)$, we conclude that the convex set $\partial g^{*}(t, 0)$ is nonempty and compact for every $t \in\left[0, r_{\Omega}\right)$. Moreover, a simple computation shows that

$$
\partial g^{*}(t, 0)=\partial f^{*}(\alpha(t)), \quad \forall t \in\left[0, r_{\Omega}\right) .
$$

The following lemma will be used in order to prove the Lipschitz regularity of the minimizers of $J$ in $\mathcal{W}(\Omega)$. 
Lemma 4. Let $g:\left[0, r_{\Omega}\right] \times \mathbb{R} \rightarrow \mathbb{R} \cup\{\infty\}$ be the function defined in 22, where $f$ satisfies the assumptions of Theorem 2 Then there exists a positive constant $C$ with the following property. If $\xi:\left[0, r_{\Omega}\right] \rightarrow \mathbb{R}$ is a measurable selection of the multifunction $t \mapsto \partial g^{*}(t, 0)$, then $|\xi(t)| \leq C$ for a.e. $t \in\left[0, r_{\Omega}\right]$.

Proof. Denote by

$$
M_{n}=\frac{1}{n}\left(\frac{|\Omega|}{v_{n}}\right)^{1 / n}
$$

the constant appearing in (11). From $[10$ we deduce that the open interval $(-M, M)$ is contained in the essential domain of $f^{*}$ (that is, $f^{*}$ is finite in that interval). Since $M>M_{n}$ by assumption (11), and $0<\alpha(t) \leq M_{n}$ by (14), from the monotonicity of the subgradient we know that $|\xi| \leq\left(f^{*}\right)_{+}^{\prime}\left(\bar{M}_{n}\right)<\infty$ for every $\xi \in \partial f^{*}(\alpha(t))$. The conclusion now follows by choosing $C=\left(f^{*}\right)_{+}^{\prime}\left(M_{n}\right)$.

Now we are in a position to prove Theorem 2 .

Proof of Theorem 2. Let $g:\left[0, r_{\Omega}\right] \times \mathbb{R} \rightarrow \mathbb{R} \cup\{\infty\}$ be the function defined in $[22$, and let $\mathcal{K}$ be the set defined in 16 . From the discussion above, it is enough to prove that the functional

$$
F(\phi)=\int_{0}^{r_{\Omega}} g\left(t, \phi^{\prime}(t)\right) d t, \quad \phi \in \mathcal{K}
$$

admits minimizers, and $\phi_{0} \in \mathcal{K}$ is a minimizer of $F$ if and only if

$$
\phi^{\prime}(t) \in \partial g^{*}(t, 0), \quad \text { a.e. } t \in\left[0, r_{\Omega}\right] .
$$

We have already shown that the multifunction

$$
t \mapsto \partial g^{*}(t, 0)=\partial f^{*}(\alpha(t)), \quad t \in\left[0, r_{\Omega}\right],
$$

has nonempty, compact convex values for every $t \in\left[0, r_{\Omega}\right)$. Moreover, from Lemma 4 if $\xi(t)$ is a measurable selection of that multifunction, then $\xi \in L^{\infty}\left(0, r_{\Omega}\right)$. Hence, the function

$$
\phi_{0}(t):=\int_{0}^{t} \xi(s) d s
$$

belongs to $\mathcal{K} \cap W^{1, \infty}\left(0, r_{\Omega}\right)$. Let us show that $\phi_{0}$ is a minimizer of $F$ in $\mathcal{K}$. Since $\phi_{0}^{\prime}(t)=$ $\xi(t) \in \partial g^{*}(t, 0)$, we deduce that $0 \in \partial g\left(t, \phi_{0}^{\prime}(t)\right)$ for a.e. $t \in\left[0, r_{\Omega}\right]$, so that

$$
F(\phi)-F\left(\phi_{0}\right)=\int_{0}^{r_{\Omega}}\left[g\left(t, \phi^{\prime}(t)\right)-g\left(t, \phi_{0}^{\prime}(t)\right)\right] d t \geq 0
$$

for every $\phi \in \mathcal{K}$.

Conversely, let $\phi_{0} \in \mathcal{K}$ be a minimizer of $F$ in $\mathcal{K}$. Let $\xi(t)$ be a measurable selection of the multifunction (24), and define $\phi(t)=\int_{0}^{t} \xi(s) d s, t \in\left[0, r_{\Omega}\right]$. From the first part of the proof, $\phi$ is a minimizer of $F$, so that $F(\phi)=F\left(\phi_{0}\right)$. Moreover, $\phi^{\prime}(t)$ is a minimum point of $g(t, \cdot)$ for a.e. $t \in\left[0, r_{\Omega}\right]$, so that

$$
g\left(t, \phi^{\prime}(t)\right)-g\left(t, \phi_{0}^{\prime}(t)\right) \leq 0, \quad \text { a.e. } t \in\left[0, r_{\Omega}\right]
$$


Since $F(\phi)=F\left(\phi_{0}\right)$, we must have

$$
g\left(t, \phi^{\prime}(t)\right)=g\left(t, \phi_{0}^{\prime}(t)\right), \quad \text { a.e. } t \in\left[0, r_{\Omega}\right] .
$$

Hence $\phi_{0}^{\prime}(t)$ must be a minimum point of $g(t, \cdot)$ for a.e. $t$, that is, 23 holds.

Remark 5. From the proof of Theorem 2 it is clear that a function $u_{0}(x)=\phi_{0}\left(d_{\Omega}(x)\right)$, $\phi_{0} \in \mathcal{K}$, is a minimizer of $J$ in $\mathcal{W}(\Omega)$ if and only if $\phi_{0}$ satisfies the Euler-Lagrange inclusion

$$
\phi_{0}^{\prime}(t) \in \partial f^{*}(\alpha(t)), \quad \text { a.e. } t \in\left[0, r_{\Omega}\right],
$$

where $\alpha$ is the function defined in $(9)$. Since $\alpha$ is strictly positive in $\left[0, r_{\Omega}\right)$, and $\partial f^{*}(p) \subset$ $[0, \infty)$ for every $p>0$, the differential inclusion 25 implies that $\phi_{0}^{\prime} \geq 0$ almost everywhere.

The last inequality can also be deduced directly from the fact that $\phi_{0}$ is a minimizer of the functional $F$ defined in (19). Namely, consider the function

$$
\phi_{1}(t)=\int_{0}^{t}\left|\phi_{0}^{\prime}(s)\right| d s, \quad t \in\left[0, r_{\Omega}\right] .
$$

Since $\phi_{0} \in \mathcal{K}$, it is easy to check that also $\phi_{1} \in \mathcal{K}$, and $0 \leq \phi_{1}^{\prime}(t)=\left|\phi_{0}^{\prime}(t)\right|$ for a.e. $t \in\left[0, r_{\Omega}\right]$. Then $\phi_{1}(t) \geq \phi_{0}(t)$ for every $t \in\left[0, r_{\Omega}\right]$. Assume by contradiction that the set $E=\left\{t \in\left[0, r_{\Omega}\right] ; \phi_{0}^{\prime}(t)<0\right\}$ has positive Lebesgue measure. In this case, $\phi_{1}>\phi_{0}$ on some interval. Hence $F\left(\phi_{1}\right)<F\left(\phi_{0}\right)$, in contradiction with the fact that $\phi_{0}$ is a minimizer of $F$.

Remark 6. Assume that $f$ satisfies (F1)-(F3), and extend $f$ over all $\mathbb{R}$ by setting $f(s)=$ $f(|s|)$ when $s<0$. We remark that (F3) is equivalent to the differentiability of this extension at $s=0$ (where we have $f^{\prime}(0)=0$ ). Hence $f$ is differentiable everywhere in $(-b, b)$. Under this differentiability assumption, the inclusion 25 can be written in the equivalent form

$$
\alpha(t)=f^{\prime}\left(\phi_{0}^{\prime}(t)\right), \quad \text { a.e. } t \in\left[0, r_{\Omega}\right]
$$

\section{Validity of the Euler-Lagrange equation}

The aim of this section is to establish a result concerning the validity of the EulerLagrange equation associated to the minimum problem (5), for minimizers belonging to the space $\mathcal{W}(\Omega)$.

Theorem 3. Let $\Omega$ be a smooth domain in $\mathbb{R}^{n}$, let $f$ satisfy (F1)-(F3), and let $u_{0} \in$ $\mathcal{W}(\Omega)$ be a minimizer of $J$ in $W_{0}^{1,1}(\Omega)$. Then $u_{0}$ satisfies the Euler-Lagrange equation

$$
\int_{\Omega}\left[f^{\prime}\left(\left|D u_{0}(x)\right|\right)\left\langle D d_{\Omega}(x), D \varphi(x)\right\rangle-\varphi(x)\right] d x=0, \quad \forall \varphi \in C_{0}^{\infty}(\Omega) .
$$


Proof. As a first step, let us prove that

$$
\lim _{\epsilon \rightarrow 0} \int_{\Omega} \frac{f\left(\left|D u_{0}+\epsilon D \varphi\right|\right)-f\left(\left|D u_{0}\right|\right)}{\epsilon} d x=\int_{\Omega} f^{\prime}\left(\left|D u_{0}\right|\right)\left\langle D d_{\Omega}, D \varphi\right\rangle d x
$$

for every $\varphi \in C_{0}^{\infty}(\Omega)$. Since $u_{0}$ is also a minimizer of $J$ in $\mathcal{W}(\Omega)$, from Theorem 2 we know that $u_{0} \in W^{1, \infty}(\Omega)$, hence there exists a positive constant $C_{0}$ such that

$$
\left|D u_{0}(x)\right| \leq C_{0}<b, \quad \text { a.e. } x \in \Omega \text {. }
$$

Now, let $\varphi$ be a fixed function in $C_{0}^{\infty}(\Omega)$. From 28 there exists $\epsilon_{0}>0$ such that

$$
\left|D u_{0}(x)+\epsilon D \varphi(x)\right| \leq C<b, \quad \text { a.e. } x \in \Omega \text {, }
$$

for every $|\epsilon| \leq \epsilon_{0}$. From the mean value theorem, for every such $\epsilon$ there exists a function $\theta_{\epsilon}$ such that

$$
\frac{f\left(\left|D u_{0}+\epsilon D \varphi\right|\right)-f\left(\left|D u_{0}\right|\right)}{\epsilon}=f^{\prime}\left(\left|D u_{0}\right|+\theta_{\epsilon}\right)\left\langle D d_{\Omega}, D \varphi\right\rangle
$$

and $\left|\theta_{\epsilon}(x)\right| \leq \epsilon|D \varphi(x)|$ for every $x \in \Omega$. This last estimate, together with 29], implies that

$$
\left|\frac{f\left(\left|D u_{0}+\epsilon D \varphi\right|\right)-f\left(\left|D u_{0}\right|\right)}{\epsilon}\right| \leq f^{\prime}(C)\|D \varphi\|_{\infty}, \quad \forall|\epsilon| \leq \epsilon_{0} .
$$

On the other hand, since $f$ is a convex differentiable function, its derivative is continuous, hence

$$
\lim _{\epsilon \rightarrow 0} \frac{f\left(\left|D u_{0}+\epsilon D \varphi\right|\right)-f\left(\left|D u_{0}\right|\right)}{\epsilon}=f^{\prime}\left(\left|D u_{0}\right|\right)\left\langle D d_{\Omega}, D \varphi\right\rangle
$$

The equality (27) now follows from the Lebesgue dominated convergence theorem.

Let us prove 26. For every $\varphi \in C_{0}^{\infty}(\Omega)$ and every $\epsilon>0$, since $u_{0}$ is a minimizer of $J$ we have

$$
0 \leq \frac{J\left(u_{0}+\epsilon \varphi\right)-J\left(u_{0}\right)}{\epsilon}=\int_{\Omega} \frac{f\left(\left|D u_{0}+\epsilon D \varphi\right|\right)-f\left(\left|D u_{0}\right|\right)-\epsilon \varphi}{\epsilon} d x .
$$

Passing to the limit as $\epsilon \rightarrow 0^{+}$, from 27) we deduce that

$$
\int_{\Omega}\left[f^{\prime}\left(\left|D u_{0}\right|\right)\left\langle D d_{\Omega}, D \varphi\right\rangle-\varphi\right] d x \geq 0
$$

Since this inequality also holds if we replace $\varphi$ with $-\varphi, 26$ follows.

Remark 7. Without the assumption that $u_{0}$ depends only on the distance from $\partial \Omega$, the validity of the Euler-Lagrange equation can be established provided that $f$ satisfies suitable growth conditions. See [7] for details. 


\section{Proof of Theorem 1}

We will use the following result.

Theorem 4 (see [6]). Let $\Omega$ be a smooth domain. Then the function $v: \bar{\Omega} \rightarrow \mathbb{R}$ defined by $v(x)=0$ for every $x \in \bar{\Sigma}$ and

$$
v(x)=\int_{0}^{\tau(x)} \prod_{i=1}^{n-1} \frac{1-\left(d_{\Omega}(x)+s\right) \kappa_{i}(x)}{1-d_{\Omega}(x) \kappa_{i}(x)} d s, \quad \forall x \in \bar{\Omega} \backslash \bar{\Sigma},
$$

is continuous in $\bar{\Omega}$ and it is the unique solution to

$$
\int_{\Omega}\left[v(x)\left\langle D d_{\Omega}(x), D \varphi(x)\right\rangle-\varphi(x)\right] d x=0, \quad \forall \varphi \in C_{0}^{\infty}(\Omega) .
$$

We recall that, for every $x \in \Omega \backslash \bar{\Sigma}, \kappa_{i}(x), i=1, \ldots, n-1$, denotes the $i$-th principal curvature of $\partial \Omega$ at $\Pi(x)$, and $\tau$ is the normal distance to the cut locus defined in 8 .

Remark 8. In the case $n=2$, Theorem 4 was proven in [5].

Let us denote by $H_{1}(y)$ the mean curvature of $\partial \Omega$ at a point $y$, that is,

$$
H_{1}(y)=\frac{1}{n-1} \sum_{i=1}^{n-1} \kappa_{i}(y), \quad y \in \partial \Omega .
$$

Under the assumptions of Theorem 1 , we shall show that $H_{1}$ is constant on $\partial \Omega$, that is, every connected component $S$ of $\partial \Omega$ is a compact embedded hypersurface without boundary with constant mean curvature $H_{1}$. From a celebrated result of Aleksandrov [1], it follows that $S$ is a hypersphere of radius $R=1 / H_{1}$. Since $\Omega$ is connected, we conclude that $\Omega$ must be a ball of radius $R$.

Proof of Theorem 1 Let $u_{0}=\phi_{0} \circ d_{\Omega}$ be a minimizer of $J$, depending only on the distance from $\partial \Omega$. Under the assumptions of Theorem 1 , from Theorem 2 we find that $\phi_{0}$ is Lipschitz continuous on $\left[0, r_{\Omega}\right]$. Furthermore, the Euler-Lagrange equation

$$
\alpha(t)=f^{\prime}\left(\left|\phi_{0}^{\prime}(t)\right|\right), \quad \text { a.e. } t \in\left[0, r_{\Omega}\right],
$$

holds, where $\alpha:\left[0, r_{\Omega}\right] \rightarrow \mathbb{R}$ is the function defined in $(9)$ (see Remark 6 ).

From Theorem 3 we know that $u_{0}$ satisfies the Euler-Lagrange equation (26). On the other hand, from Theorem 4 we deduce that $v(x)=f^{\prime}\left(\left|D u_{0}(x)\right|\right)$. From 33], we then have

$$
v(x)=\alpha\left(d_{\Omega}(x)\right), \quad \text { a.e. } x \in \Omega .
$$

More precisely, from the continuity of $v$ it follows that $v$ is constant on the level sets $\partial \Omega_{t}$ of the distance $d_{\Omega}$, for every $t \in\left[0, r_{\Omega}\right]$.

Let $x \in \bar{\Sigma}$, that is, $v(x)=0$, and let $t=d_{\Omega}(x)$. From the discussion above we have $v(y)=0$ for every $y \in \partial \Omega_{t}$, that is, $\partial \Omega_{t} \subseteq \bar{\Sigma}$. 
We claim that $t=r_{\Omega}$. Assume by contradiction that there exists a point $z \in \Omega$ with $d_{\Omega}(z)>t$, and let $y \in \Pi(z)$. The function $d_{\Omega}$ is differentiable at any point of the line segment $(y, z)$ (see [2]), which is in contradiction with the fact that $(y, z)$ must intersect $\partial \Omega_{t}$, that is, $(y, z)$ must contain at least one singular point of $d_{\Omega}$.

We have thus proven that

$$
x \in \bar{\Sigma} \Leftrightarrow d_{\Omega}(x)=r_{\Omega} .
$$

As a consequence, $d_{\Omega}$ is regular on the set $\left\{x \in \Omega ; 0<d_{\Omega}(x)<r_{\Omega}\right\}$, and then the distance to the cut locus is $\tau(y)=r_{\Omega}$ for every $y \in \partial \Omega$. From the explicit representation (30) of $v$, for every $t \in\left[0, r_{\Omega}\right)$ we have

$$
v(y)=\alpha(t)=\int_{t}^{r_{\Omega}} \prod_{i=1}^{n-1} \frac{1-s \kappa_{i}(y)}{1-t \kappa_{i}(y)} d s, \quad \forall y \in \partial \Omega_{t} .
$$

From this formula we deduce that the function $\alpha$ is of class $C^{\infty}$ on $\left[0, r_{\Omega}\right)$. By a direct computation we get

$$
\alpha^{\prime}(t)=-1+\sum_{j=1}^{n-1} \int_{t}^{r_{\Omega}}\left(\prod_{i=1}^{n-1} \frac{1-s \kappa_{i}(y)}{1-t \kappa_{i}(y)}\right) \cdot \frac{\kappa_{j}(y)}{1-t \kappa_{j}(y)} d s
$$

for every $t \in\left[0, r_{\Omega}\right)$ and $y \in \partial \Omega_{t}$. Evaluating this derivative at $t=0$ we obtain

$$
\alpha^{\prime}(0)=-1+\alpha(0) \sum_{j=1}^{n-1} \kappa_{j}(y)=-1+(n-1) \alpha(0) H_{1}(y), \quad \forall y \in \partial \Omega,
$$

so that $H_{1}$ is constant on $\partial \Omega$. From a result of Aleksandrov [1] we conclude that the connected set $\Omega$ is a ball.

Remark 9. The term $\kappa_{j}(y) /\left(1-t \kappa_{j}(y)\right)$, appearing in the integral in 34 , is the $j$-th principal curvature of the set $\partial \Omega_{t}$ at $y$ (see [16]).

Remark 10. The fact that $d_{\Omega}$ is regular on $\left\{x \in \Omega ; 0<d_{\Omega}(x)<r_{\Omega}\right\}$ alone is not enough to conclude that $\Omega$ is a ball. For example, the set $\Omega=B_{R}(0) \backslash \bar{B}_{r}(0), 0<r<R$, is a connected set with $C^{\infty}$ boundary, with inradius $r_{\Omega}=(R-r) / 2$, and the singular set $\Sigma$ coincides with $\partial B_{(r+R) / 2}(0)=\left\{x \in \Omega ; d_{\Omega}(x)=r_{\Omega}\right\}$.

Among convex sets, if we relax the assumption on the regularity of the boundary, an example can be constructed in the following way. Let $\Sigma \subset \mathbb{R}^{n}$ be a nonempty compact convex set without interior points (in the language of convex geometry, its dimension must be at most $n-1)$. Let $r>0$, and define $\Omega=\bigcup_{x \in \Sigma} B_{r}(x)$. Then $\Omega$ is an open convex set with inradius $r_{\Omega}=r$, and the singular set of $d_{\Omega}$ coincides with $\left\{x \in \Omega ; d_{\Omega}(x)=r_{\Omega}\right\}$ $=\Sigma$. 
Remark 11. Let us define the $i$-th order mean curvature $H_{i}$ of $\partial \Omega$ to be the elementary symmetric polynomial of degree $i$ in the principal curvatures $\kappa_{1}, \ldots, \kappa_{n-1}$ normalized by the following identity:

$$
\prod_{i=1}^{n-1}\left(1+\kappa_{i} t\right)=\sum_{i=0}^{n-1}\left(\begin{array}{c}
n-1 \\
i
\end{array}\right) H_{i} t^{i} .
$$

It is easily seen that $H_{0} \equiv 1$ and $H_{1}$ is the mean curvature, defined in 32 . Computing the first $n-1$ derivatives of $\alpha$ at $t=0$ we can prove that $H_{1}, \ldots, H_{n-1}$ are constant on $\partial \Omega$ (it is clear that, a posteriori, this is a consequence of the fact that $\Omega$ is a ball). For example, if $n \geq 3$, the second derivative of $\alpha$ is given by

$$
\begin{aligned}
\alpha^{\prime \prime}(t)= & -\sum_{j=1}^{n-1} \frac{\kappa_{j}(y)}{1-t \kappa_{j}(y)} \\
& +\sum_{j, h=1}^{n-1} \int_{t}^{r_{\Omega}}\left(\prod_{i=1}^{n-1} \frac{1-s \kappa_{i}(y)}{1-t \kappa_{i}(y)}\right) \cdot \frac{\kappa_{j}(y) \kappa_{h}(y)}{\left(1-t \kappa_{j}(y)\right)\left(1-t \kappa_{h}(y)\right)} d s
\end{aligned}
$$

for every $t \in\left[0, r_{\Omega}\right)$ and $y \in \partial \Omega_{t}$, hence

$$
\alpha^{\prime \prime}(0)=-(n-1) H_{1}(y)+(n-1)(n-2) H_{2}(y) \alpha(0), \quad \forall y \in \partial \Omega .
$$

Since $H_{1}$ is constant on $\partial \Omega$, we deduce that also $H_{2}$ is constant on $\partial \Omega$.

\section{References}

[1] Aleksandrov, A. D.: Uniqueness theorems for surfaces in the large. I, II. Amer. Math. Soc. Transl. 21, 341-388 (1962)

[2] Bardi, M., Capuzzo-Dolcetta, I.: Optimal Control and Viscosity Solutions of HamiltonJacobi-Bellman Equations. Birkhäuser, Boston (1997) Zbl 0890.49011 MR 1484411

[3] Brock, F., Prajapat, J.: Some new symmetry results for elliptic problems on the sphere and in Euclidean space. Rend. Circ. Mat. Palermo 49, 445-462 (2000) Zbl 1008.35002 MR 1809087

[4] Burago, Yu. D., Zalgaller, V. A.: Geometric Inequalities. Springer, Berlin (1988) Zbl 0633.53002 MR 0936419

[5] Cannarsa, P., Cardaliaguet, P.: Representation of equilibrium solutions to the table problem for growing sandpiles. J. Eur. Math. Soc. 6, 435-464 (2004) Zbl pre02139679 MR 2094399

[6] Cannarsa, P., Cardaliaguet, P., Crasta, G., Giorgieri, E.: A boundary value problem for PDE. Mathematical models of mass transfer: representation of solutions and applications. Calc. Var. Partial Differential Equations, to appear

[7] Cellina, A.: On the validity of the Euler-Lagrange equation. J. Differential Equations 171, 430-442 (2001) Zbl 1015.49018 MR 1818657

[8] Crasta, G.: Existence, uniqueness and qualitative properties of minima to radially symmetric non-coercive non-convex variational problems. Math. Z. 235, 569-589 (2000) Zbl 0965.49003 MR 1800213

[9] Crasta, G.: Variational problems for a class of functionals on convex domains. J. Differential Equations 178, 608-629 (2002) Zbl 1019.49020 MR 1879839 
[10] Crasta, G.: Estimates for the energy of the solutions to elliptic Dirichlet problems on convex domains. Proc. Roy. Soc. Edinburgh Sect. A 134, 89-107 (2004) Zbl pre02114666 MR 2039904

[11] Crasta, G., Fragalà, I., Gazzola, F.: A sharp upper bound for the torsional rigidity of rods by means of web functions. Arch. Rat. Mech. Anal. 164, 189-211 (2002) Zbl 1021.74020 MR 1930391

[12] Crasta, G., Fragalà, I., Gazzola, F.: On the role of energy convexity in the web function approximation. NoDEA Nonlinear Differential Equations Appl. 12, 93-109 (2005) MR 2138936

[13] Damascelli, L., Pacella, F.: Monotonicity and symmetry results for $p$-Laplace equations and applications. Adv. Differential Equations 5, 1179-1200 (2000) Zbl 1002.35045 MR 1776351

[14] Evans, L. C., Gariepy, R. F.: Measure Theory and Fine Properties of Functions. CRC Press, Boca Raton (1992) Zbl 0804.28001 MR 1158660

[15] Federer, H.: Curvature measures. Trans. Amer. Math. Soc. 93, 418-491 (1959) Zbl 0089.38402 MR 0110078

[16] Feldman, M.: Variational evolution problems and nonlocal geometric motion. Arch. Rat. Mech. Anal. 146, 221-274 (1999) Zbl 0955.49025 MR 1720391

[17] Itoh, J., Tanaka, M.: The Lipschitz continuity of the distance function to the cut locus. Trans. Amer. Math. Soc. 353, 21-40 (2001) Zbl 0971.53031 MR 1695025

[18] Li, Y.Y., Nirenberg, L.: The distance function to the boundary, Finsler geometry and the singular set of viscosity solutions of some Hamilton-Jacobi equations. Comm. Pure Appl. Math. 58, 85-146 (2005) Zbl pre02126519 MR 2094267

[19] Serrin, J.: A symmetry problem in potential theory. Arch. Rat. Mech. Anal. 43, 304-318 (1971) Zbl 0222.31007 MR 0333220

[20] Vornicescu, M.: A variational problem on subsets of $\mathbb{R}^{n}$. Proc. Roy. Soc. Edinburgh Sect. A 127, 1089-1101 (1997) Zbl 0920.49002 MR 1475648

[21] Ziemer, W. P.: Weakly Differentiable Functions. Springer, New York (1989) Zbl 0692.46022 MR 1014685 